\title{
Clustering in a highly hydrogenated diamondlike carbon determined using fluctuation electron microscopy and phenomenological atomistic simulations
}

\author{
A. C. Y. Liu* \\ Materials Science Division, Argonne National Laboratory, Illinois 60439-4845, USA \\ R. Arenal \\ Materials Science Division, Argonne National Laboratory, Illinois 60439-4845, USA \\ and Laboratoire d'Etude des Microstructures, ONERA-CNRS, 92322 Châtillon, France \\ Xidong Chen \\ Electron Microscopy Center, Argonne National Laboratory, Illinois 60439-4845, USA \\ and Cedarville University, Cedarville, Ohio 45314, USA \\ (Received 1 July 2007; published 4 September 2007)
}

\begin{abstract}
We compare fluctuation electron microscopy data to simulations from phenomenological atomic models and demonstrate a strong correspondence between some features in the experimental data and certain atomic configurations. This allows the nature of atomic clustering in a highly hydrogenated diamondlike carbon to be determined more closely. We compare the structural information garnered from fluctuation electron microscopy and Raman spectroscopy for a particular diamondlike carbon and find consistency between the two techniques in the region where their information overlaps.
\end{abstract}

DOI: 10.1103/PhysRevB.76.121401

PACS number(s): 61.43.Er, 61.43.Bn, 63.50.+x

Amorphous carbons, or diamondlike carbons (DLCs), are becoming the material of choice for protective coatings due to their hardness, chemical inertness, transparency, low coefficient of friction, and wear resistance. ${ }^{1}$ The three bonding hybridizations available to carbon $\left(s p^{1}, s p^{2}\right.$ or $\pi$, and $s p^{3}$ or $\sigma$ ) and the ability to introduce hydrogen into the amorphous network allow DLCs to have their properties tuned to be more graphitic, diamondlike, or polymeric. ${ }^{2}$ However, the properties of a given DLC are not determined uniquely by the ratio of the carbon hybridizations and the amount of hydrogen incorporated. ${ }^{3}$ Clustering of bonded carbon atoms in the DLC must also be taken into account; as an example, the clustering of the $s p^{2}$ phase plays the dominant role in determining the optical and electronic properties. ${ }^{4}$ This added degree of freedom gives rise to hysteresis in the "amorphization trajectory" of the material whereby one DLC may have the same absolute fraction of carbon bonding as another DLC, but different degrees of clustering. ${ }^{5}$

Raman spectroscopy has led the way in making these finer distinctions in DLCs, ${ }^{4,5}$ hydrogenated DLCs,${ }^{6}$ and amorphous carbon nitrides. ${ }^{7}$ The way that Raman spectroscopy accesses this structural information is via the vibrational density of states of the material. This gives rise to a resonantly enhanced sensitivity to the $s p^{2}$-bonded carbon and a Raman spectrum dominated by the structure of the $s p^{2}$-bonded carbon. ${ }^{4}$ Specifically, two main features may be found in the experimental Raman spectrum of an amorphous carbon, the $D$ peak at $\sim 1350 \mathrm{~cm}^{-1}$ and the $G$ peak at $\sim 1500-1630 \mathrm{~cm}^{-1}$. The $D$ and $G$ peaks, respectively, correspond to a breathing mode requiring the presence of aromatic rings and an in-plane bond-stretching mode that may occur at any $s p^{2}$ site regardless of whether it is in a ring. ${ }^{4}$ Thus the ratio of the intensities of the $D$ and $G$ peaks, $I(D) / I(G)$, gives some measure of the ordering of the $s p^{2}$ phase, as the $D$ peak will increase in intensity as the number of rings with delocalized $\pi$ bonds increases. At a set exciting wavelength, the position of the $G$ peak increases as the cluster size of the $s p^{2}$ phase changes from rings to chains to dimers. ${ }^{4}$ The cluster size probed is determined by the exciting radiation used, as a smaller wavelength will resonate with smaller cluster sizes. Thus, dispersion of features in the spectrum as a function of the Raman exciting wavelength yields even greater insight. For example, the dispersion of the $G$ peak position, $\operatorname{Disp}(G)$, gives an idea of the distribution of $s p^{2}$ chain sizes. If no $s p^{2}$ bonds exist in chains and only perfect rings are present, then $\operatorname{Disp}(G)=0$. At any wavelength, the full width at half maximum of the $G$ peak, $\operatorname{FWHM}(G)$, gives some measure of cluster disorder for that particular cluster size. At this point the interpretation of the features in the Raman spectrum is largely qualitative; however, estimates of the ratio of $s p^{2}$ to $s p^{3}$ bonding and $s p^{2}$ cluster size may be obtained through comparison to similar samples and by imposing continuity between the amorphous carbon and nanocrystalline (nc) graphite regimes. ${ }^{4}$

Here we present simulations of fluctuation electron microscopy (FEM) data from representative clusters that may be present in an amorphous carbon. FEM measures the variation in scattered intensity from volumes of material with lateral extent comparable to the length scale for medium-range atomic order (MRO). This intensity variance $V$ as a function of scattering vector $k$ is related to the pair-pair correlation function in the material and hence gives a strong signature of the regions of correlated structure, or clusters, that are present. $^{8}$ As it relies on electron scattering, FEM accesses structural information directly via the phase shifts undergone by electrons scattered by atoms at different positions. Thus, in contrast to Raman spectroscopy, FEM has equal sensitivity to $s p^{2}$ - and $s p^{3}$-bonded carbon configurations, as the only modulation to the scattering is in the form of the atomic scattering factor, which is virtually the same for electron 
scattering from $s p^{2}$ - and $s p^{3}$-bonded carbon atoms. FEM also promises to be more sensitive to highly distorted configurations, as the perfect bonding that gives rise to the vibrational modes does not have to be present. Although FEM accesses atomic correlations beyond two-body correlations and therefore probes structural information inaccessible to diffraction, the $V(k)$ cannot be directly inverted to yield the atomic correlation functions. As a consequence, quantification of the MRO from FEM remains a challenge. ${ }^{8}$ In this work we demonstrate that considerable progress can be made toward quantification by simulating $V(k)$ from phenomenological atomic models using an analytical expression.

Figure 1(a) displays an experimental $V(k)$ curve obtained from a low density, $\sim 80 \% s p^{2}$-bonded hydrogenated DLC made by plasma-enhanced chemical vapor deposition. ${ }^{3}$ Such films contain a high proportion of hydrogen, but unexpectedly low fractions of $s p^{3}$ bonding. We denote them hydrogenated DLCs in line with the classifications of Casiraghi et al. ${ }^{6}$ Such materials have been found to display strikingly low coefficients of friction; ${ }^{9}$ the friction properties have been correlated with the short- and medium-range atomic order as a function of depth in the film. ${ }^{3}$ This particular experimental curve is acquired from the bulk of a film grown with a source gas composition of $75 \% \mathrm{H}_{2}$ and $25 \% \mathrm{CH}_{4}$ that also manifests the lowest coefficient of friction in the set previously studied. ${ }^{3}$ FEM was performed in tilted dark-field mode using an automated acquisition system on a Jeol JEM-4000 EXII. Details of the specimen preparation and data analysis have been described elsewhere. ${ }^{3}$ Briefly, cross-sectional samples were prepared by mechanical polishing in a wedge geometry and subsequent low-energy ion milling for short times. ${ }^{3}$ Dark-field images were treated with a Wiener filter prior to calculation of the average intensity and $V(k) .{ }^{10,3}$ Error bars represent the standard error from measurements on ten different areas. The $V(k)$ curve has three peaks that have been associated with clusters with local graphitic bonding $\left(2.8 \mathrm{~nm}^{-1}\right)$ and local diamondlike bonding (5 and $\left.8.8 \mathrm{~nm}^{-1}\right){ }^{3,10} \mathrm{In}$ accord with these assignments, the $V(k)$ curves from highly tetrahedral amorphous carbon, or DLC with a large $(\sim 80 \%) s p^{3}$-bonded carbon fraction, lack the feature at $2.8 \mathrm{~nm}^{-1}$. $^{10}$

Recent work has extended the Debye equation that describes the scattered intensity from a random assembly of atoms to obtain a simple expression for the intensity variance. ${ }^{11,12}$ In this expression the intensity variance is a function of the scattering vector $k$, the atomic scattering factor $f(k)$, and also the sum of the separations of two pairs of atoms, $\mathbf{r}_{i j}+\mathbf{r}_{k l}$. This direct dependence on pair-pair atomic correlations gives rise to the extreme sensitivity of this technique to clustering. Here we apply this Debye formulation of the intensity variance to examine the FEM signatures of different carbon clusters. This technique for calculating the variance is ideal for looking at small structural motifs rather than large atomic models, as it provides a variance that is the equally weighted sum of the variance for all possible cluster orientations with respect to the incident electron beam. This is equivalent to filling space with randomly oriented clusters separated sufficiently to suppress intercluster correlations, or constructing a larger atomic model in which the cluster is the a

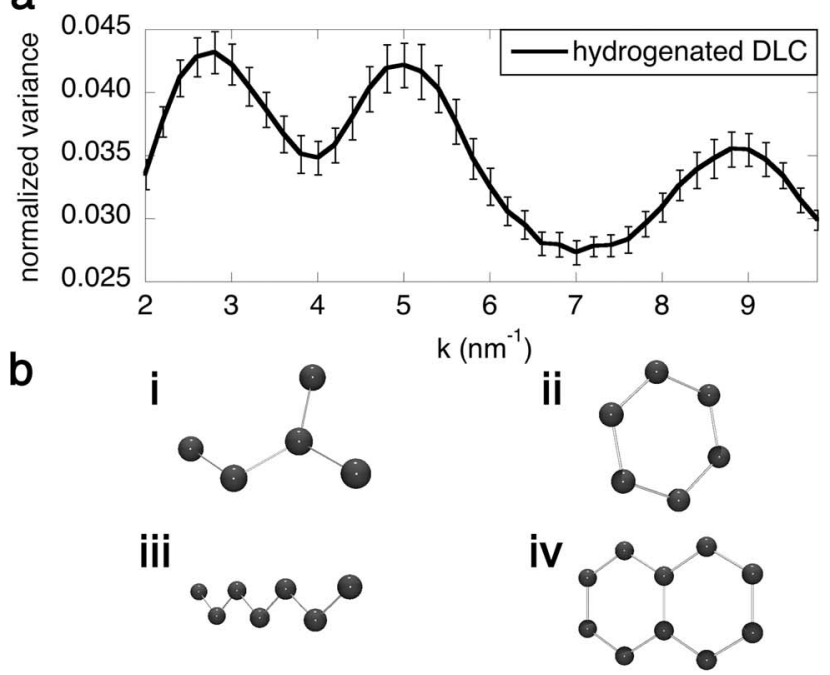

C

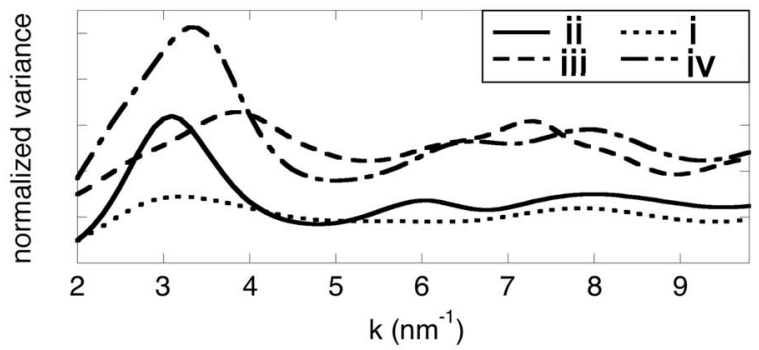

d

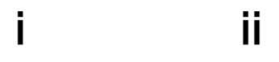

ii

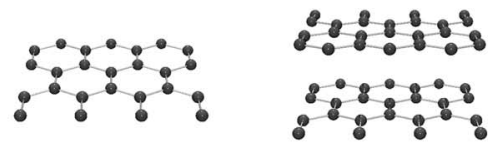

iii

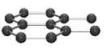

$\because: 3$

$2:$

e

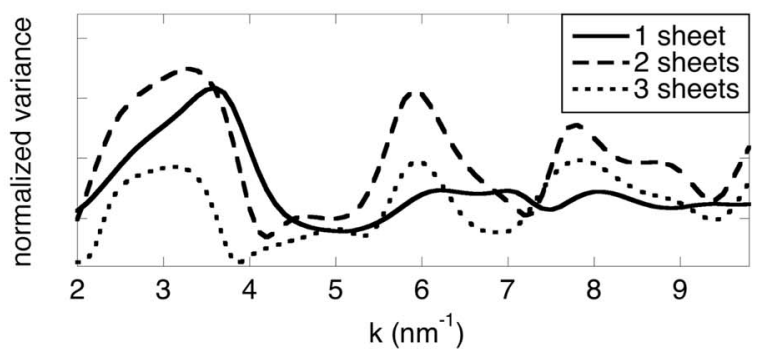

FIG. 1. (a) Experimental $V(k)$ curve from a hydrogenated, highly $s p^{2}$-bonded DLC. (b) Fragments of graphitic structure used to generate simulated $V(k)$ curves in (c). (d) Graphene sheet structures used to generate simulated $V(k)$ curves in (e).

dominant structurally correlated motif. We note that we will consider only carbon configurations; the small scattering power of hydrogen means that the FEM will be 5-125 times less sensitive to structural correlations involving a hydrogen atom than to purely carbon ones. ${ }^{13}$ As we are only considering correlations in the carbon atoms, and using monatomic clusters, the atomic scattering factor $f(k)$ will cancel in the expression for the normalized intensity variance $V(k)$. We also use fragments of crystalline structures smaller than the resolution of the microscope for a typical FEM experiment as this parameter determines the length scale over which structural correlations may be detected.

Turning first to clusters with graphitic local bonding, we 
a

b
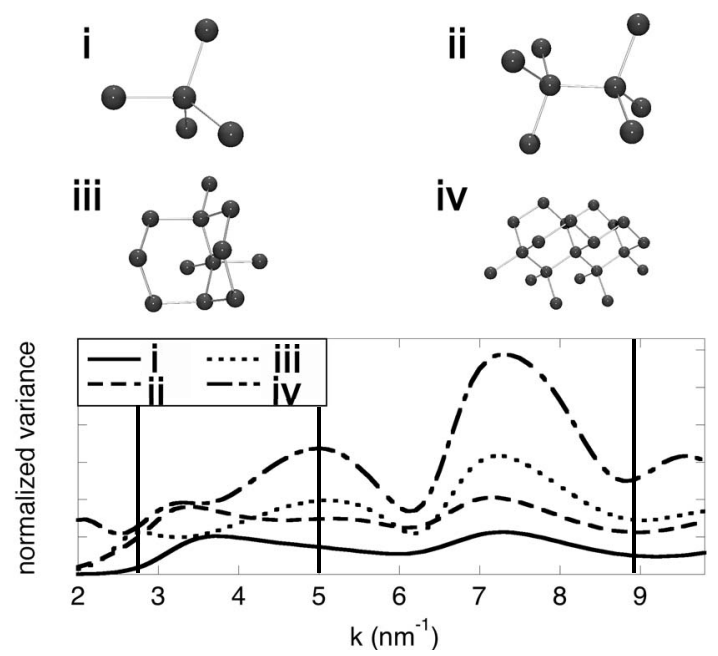

FIG. 2. (a) Fragments of diamond structure used to generate simulated $V(k)$ curves in (b).

simulated the $V(k)$ from small fragments of the graphite structure. The fragments consist of a broken ring, a chain, and full and double rings as shown in Fig. 1(b). The simulations are shown in part (c) of the same figure. It is readily seen that incomplete rings [structures (b) (i) and (iii)] do not give rise to the peak in the variance at $2.8 \mathrm{~nm}^{-1}$ that is observed in the data and attributed to graphitic local bonding. Complete rings such as those in structures (b) (ii) and (iv) do yield a prominent peak at this position. Thus the simulations suggest that complete aromatic rings are present in this particular DLC. The other configurations of broken ring and chain may also exist, but their broad peaks add to the magnitude of the variance in a way that is harder to uniquely attribute. In Fig. 1(e) we show variance simulations from graphitic fragments comprised of one, two, and three graphene sheets as illustrated in Fig. 1(d) (i)-(iii). The single sheet has a very prominent peak at low $k$, broadly consistent with observation, but shifted slightly to higher wave vector. The simulations from the fragments composed of multiple sheets also possess this low- $k$ peak, but additionally have peaks in the $V(k)$ curve at higher wave vector that do not correspond to any of the peaks in the data. Thus our initial survey of signatures in the FEM from graphitic fragments suggests that the $s p^{2}$-bonded carbon is present in rings, but that greater association between these rings in layered structures is not in evidence.

We conducted similar studies of the diamond structure as displayed in Fig. 2. Figure 2(a) of this figure shows the test structures, which range from (i) the single, isolated tetrahedron to (ii) the double tetrahedron with dihedral angle preserved, to structures with (iii) two and (iv) four sixmembered rings with the typical chair conformation. Examining the simulations in part (b) of this figure, we see that the tetrahedra do not possess peaks in their simulated $V(k)$ at the positions found in the data (indicated in the plot with solid vertical lines in this and subsequent figures). The simulated $V(k)$ 's of the six-membered ring configurations do have a peak at $5 \mathrm{~nm}^{-1}$ in common with the data, but have a large peak at higher wave vector with no match in the ex- a

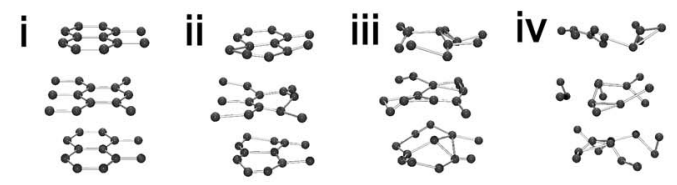

b

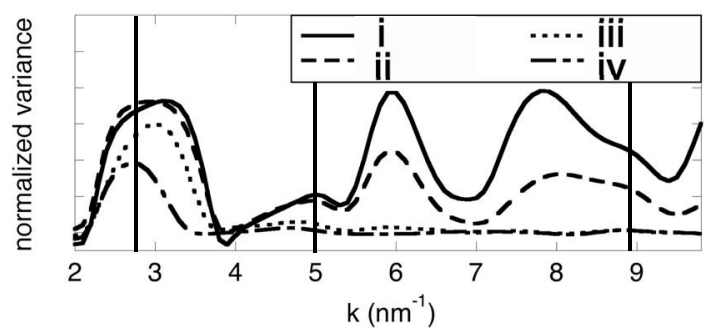

C

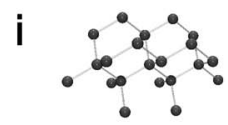

ii

iii
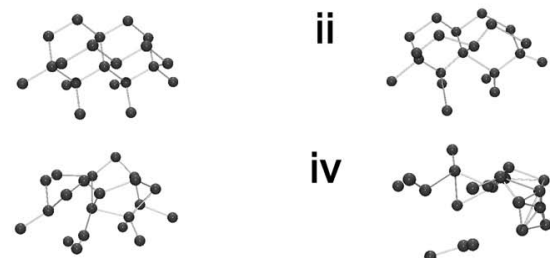

iv

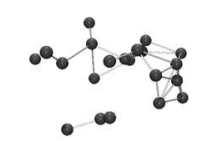

d

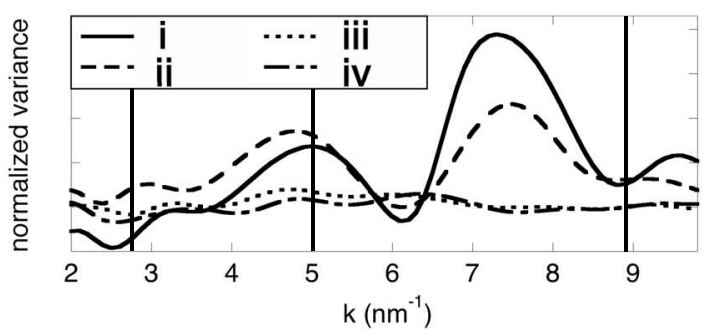

FIG. 3. (a) Graphitic models with progressively randomized atomic positions (i)-(iv) used to simulate $V(k)$ curves displayed in (b). (a) Diamondlike models with progressively randomized atomic positions (i)-(iv) used to simulate $V(k)$ curves displayed in (d).

perimental curve. These observations seem to suggest that the $s p^{3}$ phase may be organized in rings with the chair conformation in this DLC, but that a systematic distortion of this structure in the amorphous phase may modulate the peak in the simulated data at $7.5 \mathrm{~nm}^{-1}$.

As we had no basis for distorting our test structures in a systematic way, we explored the simulated $V(k)$ from structures that had random displacements to their atomic positions. To randomize the atomic positions we applied separately a Gaussian noise to the atomic coordinates in each dimension with maximum values of $0.5,1.0$, and $1.5 \AA$. The randomized structures for the three-layered graphite structure and the four six-membered chair-conformed ring diamond structure are shown in order of increasing randomization in Figs. 3(a) and 3(c). The accompanying simulations are shown in Figs. 3(b) and 3(d) of this same figure. We see that randomization extinguishes the two high-wave-vector peaks in the simulated $V(k)$ of the three-layered graphite structure such that by the time the noise with maximum of $1.0 \AA$ is applied [Fig. 3(a) (iii)] the $V(k)$ has only the single peak at $2.8 \mathrm{~nm}^{-1}$. In the case of the diamond structure positional randomization to a maximum of $1.0 \AA$ also seems to act to destroy the peak magnitudes, but does not result in curves that better match the experimental data.

Raman spectroscopy was performed at room temperature 


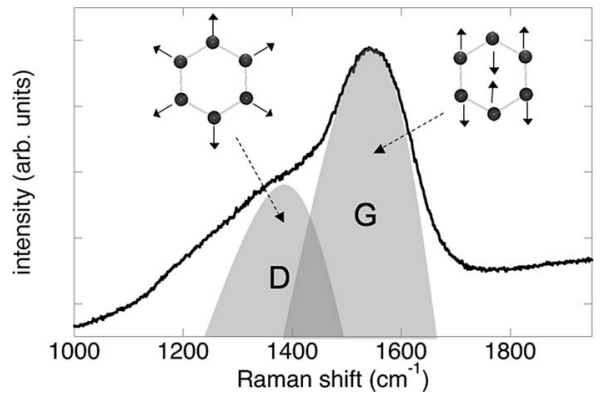

FIG. 4. Raman spectrum $(514 \mathrm{~nm})$ of the DLC studied in this paper with features corresponding to the breathing $(D)$ and stretching $(G)$ vibrational modes. We note that stretching modes can also be found in $s p^{2}$-bonded chains (Ref. 4).

in a backscattering geometry using an argon-ion laser with a wavelength of $514 \mathrm{~nm}$ and a Jobin-Yvon Labram HR800-Vis spectrometer. The laser power and dwell time were such that no damage to the specimen could be detected in the regions studied. Figure 4 displays the Raman spectrum obtained from the same DLC film studied with FEM [Fig. 1(a)]. We see that the $D$ and $G$ peaks are clearly present. As with the FEM it is not clear exactly what is the proportion of $s p^{2}$-bonded carbon in rings and in chains, although $\operatorname{disp}(G)$ does indicate that there is a finite-size distribution of these clusters. ${ }^{14}$ Using an empirical expression ${ }^{4}$ relating cluster diameter to $I(D) / I(G)$ we find the cluster diameter $L_{a} \sim 13 \AA$. This corresponds to approximately four aromatic rings per cluster, similar to the structure simulated in Fig. 1(d) (i). However, $L_{a}$ is a parameter for the in-plane cluster correlation length ${ }^{4}$ and so the cluster size estimate from Raman spectroscopy is not sensitive to the differences in the structures in Fig. 1(d), (i), (ii), and (iii).

In summary, from our simulations of the features found in
FEM data from a hydrogenated DLC we identify the $s p^{2}$-bonded carbon to be configured in aromatic rings. It appears that these rings are not associated in layers, except if the structure is so distorted that layer identification is itself compromised. The $s p^{3}$-bonded carbon in this material seems to be configured in fragments larger than a single or double tetrahedron that possess the typical six-membered ring structure in the chair conformation of diamond. However, it seems as though a systematic distortion needs to be applied to these structures to produce an exact match to the experimental data. This distorted structure may be stabilized by the presence of hydrogen, even if carbon-hydrogen configurations are not detected with as much efficiency with FEM as carbon-carbon configurations. $s p^{2}$ carbon in aromatic rings can be determined from the presence of the $D$ peak in the Raman spectrum. ${ }^{5}$ However, distorted rings without fully delocalized $\pi$ bonds can't be detected with this technique. The $\operatorname{FWHM}(G)$ and $\operatorname{Disp}(G)$ can be used to qualitatively probe for distortion in clusters and a distribution of cluster sizes. As we noted, though, Raman spectroscopy has little sensitivity to the configurations of the $s p^{3}$-bonded carbon. In contrast, we see that FEM has the potential to identify distorted structures composed of both carbon bonding types. There is particular promise in matching simulations of FEM from larger atomic models generated using for example $a b$ initio techniques to experimental curves.

This work was supported in part by the U.S. Department of Energy under Contract No. DE-AC02-06CH11357 at ANL. The TEM was conducted in the Electron Microscopy Center at ANL. We thank A. Erdemir, O. Eryilmaz, J. A. Johnson, and J. B. Woodford for providing the DLC films and participating in many lively discussions. G. Montagnac at the Ecole Normal Supérieure, Lyon, is thanked for his assistance with the Raman spectroscopy. Stimulating discussions with M. M. J. Treacy are gratefully acknowledged. *aliu@anl.gov

${ }^{1}$ J. Robertson, Mater. Sci. Eng., R. 37, 129 (2002).

${ }^{2}$ C. Casiraghi, J. Robertson, and A. C. Ferrari, Mater. Today 10, 44 (2007).

${ }^{3}$ A. C. Y. Liu, R. Arenal, D. J. Miller, X. Chen, J. A. Johnson, O. L. Eryilmaz, A. Erdemir, and J. B. Woodford, Phys. Rev. B 75, 205402 (2007).

${ }^{4}$ A. C. Ferrari and J. Robertson, Phys. Rev. B 61, 14095 (2000).

${ }^{5}$ A. C. Ferrari and J. Robertson, Phys. Rev. B 64, 075414 (2001).

${ }^{6}$ C. Casiraghi, A. C. Ferrari, and J. Robertson, Phys. Rev. B 72, 085401 (2005).

${ }^{7}$ A. C. Ferrari, S. E. Rodil, and J. Robertson, Phys. Rev. B 67, 155306 (2003).
${ }^{8}$ M. M. J. Treacy, J. M. Gibson, L. Fan, D. J. Paterson, and I. McNulty, Rep. Prog. Phys. 68, 2899 (2005).

${ }^{9}$ A. Erdemir, O. L. Eryilmaz, and G. Fenske, J. Vac. Sci. Technol. B 18, 1987 (2000).

${ }^{10}$ X. Chen, J. P. Sullivan, T. A. Freidmann, and J. M. Gibson, Appl. Phys. Lett. 84, 2823 (2004).

${ }^{11}$ W. E. McBride, D. R. McKenzie, D. G. McCulloch, D. J. H. Cockayne, and T. C. Petersen, J. Non-Cryst. Solids 351, 413 (2005).

${ }^{12}$ W. E. McBride, Ph.D. thesis, University of Sydney, 2000.

${ }^{13}$ L.-M. Peng, G. Ren, S. L. Dudarev, and M. J. Whelan, Acta Crystallogr., Sect. B: Struct. Sci. 52, 257 (1996).

${ }^{14}$ R. Arenal and A. C. Y. Liu (unpublished). 